\title{
Mean curvature flow singularities for mean convex surfaces
}

\author{
Gerhard Huisken $^{1}$, Carlo Sinestrari ${ }^{2}$
}

1 Mathematisches Institut, Universität Tübingen, Auf der Morgenstelle 10, D-72076 Tübingen, Germany (e-mail: gerhard.huisken@uni-tuebingen.de)

2 Dipartimento di Matematica, Università di Roma "Tor Vergata", Via della Ricerca Scientifica, I-00133 Roma, Italy, (e-mail: sinestra@mat.utovrm.it)

Received July 11,1997 / Accepted November 14, 1997

\begin{abstract}
We study the evolution by mean curvature of a smooth n-dimensional surface $\mathscr{M} \subset \mathbb{R}^{n+1}$, compact and with positive mean curvature. We first prove an estimate on the negative part of the scalar curvature of the surface. Then we apply this result to study the formation of singularities by rescaling techniques, showing that there exists a sequence of rescaled flows converging to a smooth limit flow of surfaces with nonnegative scalar curvature. This gives a classification of the possible singular behaviour for mean convex surfaces in the case $n=2$.
\end{abstract}

\section{Introduction}

Let $\mathscr{C}$ be a compact $n$-dimensional manifold without boundary and let $F_{0}$ : $\mathscr{C} \rightarrow \mathbb{R}^{n+1}$ be a smooth immersion of $\mathscr{\mathscr { C }}$ as a hypersurface. We want to study the evolution of $\mathscr{C}_{0}=F_{0}(\mathscr{C})$ by mean curvature flow; that is, the family of immersions $F(\cdot, t)$ satisfying

$$
\frac{\partial F}{\partial t}(p, t)=-H(p, t) \nu(p, t), \quad p \in \mathscr{M}, t \geq 0,
$$

$$
F(\cdot, 0)=F_{0},
$$

where $H(p, t)$ and $\nu(p, t)$ are the mean curvature and the outer normal respectively at the point $F(p, t)$ of the surface $\mathscr{U}_{t}=F(\cdot, t)(\mathscr{C})$. The signs are chosen such that $-H \nu=\vec{H}$ is the mean curvature vector and the mean curvature of a convex surface is positive.

It is well known that problem (1.1)-(1.2) is a quasilinear parabolic system and that the mean curvature flow is well defined up to a finite critical time $T$ at which the curvature of the surface becomes unbounded. Moreover, various geometric properties (for instance convexity or positive mean curvature) are invariant under the flow. 
The structure of the singularity of $\mathscr{M}_{t}$ when $t$ approaches the critical time has been of great interest. For instance, when the initial surface $\mathscr{\mathscr { C }}_{0}$ is convex, then as $t \rightarrow T$ the surfaces $\mathscr{C}_{t}$ become spherical and contract to a point. This result was obtained by Gage and Hamilton [5] in the case $n=1$ and by Huisken [10] when $n \geq 2$; it was then extended by Grayson [6] to arbitrary embedded curves. On the other hand, for $n \geq 2$ nonconvex surfaces may become singular without shrinking to a point also in the embedded case. Then one can analyse the behaviour near a singularity with rescaling techniques. In [11] Huisken showed that singularities having a certain maximal blow-up rate (type I singularities) are asymptotically self-similar. For surfaces of positive mean curvature the possible limiting profiles were then classified in [12]. The singularities of rotationally symmetric surfaces were described by Altschuler, Angenent and Giga [2] and by Angenent and Velazquez [3], while other rescalings of mean curvature flow in the case $n=2$ were recently studied by Ilmanen [14] and White [17].

In this paper we consider a general surface with positive mean curvature and with $n \geq 2$. Without any assumption on the blow-up rate, we prove a new a priori estimate, Theorem 3.1, showing that the scalar curvature of the surface is asymptotically nonnegative near a singularity. This implies that a sequence of rescaled flows tends to a limit flow of surfaces with nonnegative scalar curvature (Theorem 4.5). Such a property is particularly interesting for $n=2$ because then positive scalar curvature is equivalent to convexity. This leads to a classification of the possible limiting shapes both for type I and type II singularities (Corollary 4.7).

\section{Preliminaries}

In the next sections we study the mean curvature flow (1.1)-(1.2) assuming that the initial hypersurface $\mathscr{\mathscr { C }}_{0}=F_{0}(\mathscr{C}) \subset \mathbb{R}^{n+1}(n \geq 2)$ is smooth, compact without boundary and has positive mean curvature. It is well known (see [10, Theorem 8.1]) that there exists a smooth solution to problem (1.1)-(1.2) up to a critical time $T$ at which the curvature of the evolving surface becomes unbounded. This critical time is finite for any compact $\mathscr{\mathscr { O }}_{0}$, and satisfies

$$
T \leq \frac{\left(\operatorname{diam} \mathscr{M}_{0}\right)^{2}}{8 n}
$$

We recall now the equations for some geometric quantities associated with the evolving surface and other identities which we shall need in the sequel. We shall follow the notations of [10]; in particular $g=\left(g_{i j}\right)$ and $A=\left(h_{i j}\right)(i, j=1, \ldots, n)$ will denote the metric tensor and the second fundamental form on $\mathscr{N}$ induced by the immersion, while $H=\operatorname{tr}\left(h_{i j}\right)$ and $R=H^{2}-|A|^{2}$ are the mean curvature and the scalar curvature respectively. We also denote by $d \mu$ the volume element on $\mathscr{M}$. All these quantities depend on $x, t$ (where $x$ is a local coordinate on $\mathscr{M})$, but this dependence will not be written explicitly unless necessary.

Proposition 2.1. We have the evolution equations 
(i) $\frac{\partial H}{\partial t}=\Delta H+|A|^{2} H$,

(ii) $\frac{\partial|A|^{2}}{\partial t}=\Delta|A|^{2}-2|\nabla A|^{2}+2|A|^{4}$,

(iii) $\frac{\partial}{\partial t} d \mu=-H^{2} d \mu$.

Proof. See [10].

From (i) and the maximum principle we obtain that the mean curvature of our surface remains positive during the evolution.Let us now introduce, for $\eta \in \mathbb{R}$ and $\sigma \in[0,2]$, the function

$$
g_{\sigma, \eta}=\frac{|A|^{2}-(1+\eta) H^{2}}{H^{2-\sigma}} .
$$

Then we compute, using (i) and (ii) from the previous proposition,

$$
\begin{aligned}
\frac{\partial g_{\sigma, \eta}}{\partial t}= & \Delta g_{\sigma, \eta}+\frac{2(1-\sigma)}{H}\left\langle\nabla H, \nabla g_{\sigma, \eta}\right\rangle-\frac{\sigma(1-\sigma)}{H^{2}} g_{\sigma, \eta}|\nabla H|^{2} \\
& -\frac{2}{H^{4-\sigma}}\left|H \nabla_{i} h_{k l}-\nabla_{i} H h_{k l}\right|^{2}+\sigma|A|^{2} g_{\sigma, \eta}
\end{aligned}
$$

In particular, for $\sigma=0$, we find

$$
\frac{\partial}{\partial t} \frac{|A|^{2}}{H^{2}}=\Delta \frac{|A|^{2}}{H^{2}}+\frac{2}{H}\left\langle\nabla H, \nabla \frac{|A|^{2}}{H^{2}}\right\rangle-\frac{2}{H^{4}}\left|H \nabla_{i} h_{k l}-\nabla_{i} H h_{k l}\right|^{2} .
$$

By the maximum principle and by the compactness of $\mathscr{M}$ we obtain that $|A|^{2} / H^{2}$ is uniformly bounded from above by its initial data. Thus, if we call $c_{0}$ the maximum of $|A|^{2} / H^{2}$ on $\mathscr{M}_{0}$, we have

$$
|A|^{2} \leq c_{0} H^{2} \text { on } \mathscr{M}_{t}, \forall t \in[0, T[.
$$

Let us also recall the identity (see [10])

$$
\frac{1}{2} \Delta|A|^{2}=\left\langle h_{i j}, \nabla_{i} \nabla_{j} H\right\rangle+|\nabla A|^{2}+Z,
$$

where $Z=H \operatorname{tr}\left(A^{3}\right)-|A|^{4}$. The following elementary inequality will be useful to derive estimates on $Z$.

Lemma 2.2. We have $\left|\operatorname{tr}\left(A^{3}\right)\right| \leq|A|^{3}$.

Proof. If we denote by $\lambda_{1}, \ldots, \lambda_{n}$ the eigenvalues of the second fundamental form, the above inequality can be written as

$$
\left|\sum_{i=1}^{n} \lambda_{i}^{3}\right| \leq\left(\sum_{i=1}^{n} \lambda_{i}^{2}\right)^{3 / 2} .
$$

It is not restrictive to assume $|A|>0$. If we set 


$$
\mu_{i}=\frac{\left|\lambda_{i}\right|}{\left(\sum_{j=1}^{n} \lambda_{j}^{2}\right)^{1 / 2}}, \quad i=1, \ldots, n
$$

then $\left|\mu_{i}\right| \leq 1$, and therefore $\mu_{i}^{3} \leq \mu_{i}^{2}$. It follows

$$
\begin{aligned}
\left|\sum_{i=1}^{n} \lambda_{i}^{3}\right| & \leq\left(\sum_{i=1}^{n} \mu_{i}^{3}\right)\left(\sum_{j=1}^{n} \lambda_{j}^{2}\right)^{3 / 2} \\
& \leq\left(\sum_{i=1}^{n} \mu_{i}^{2}\right)\left(\sum_{j=1}^{n} \lambda_{j}^{2}\right)^{3 / 2} \\
& =\left(\sum_{j=1}^{n} \lambda_{j}^{2}\right)^{3 / 2} .
\end{aligned}
$$

\section{An estimate on the scalar curvature}

The goal of this section is to prove the following result.

Theorem 3.1. Let $\mathscr{M}_{t}, t \in[0, T$ [ be a smooth solution of the mean curvature flow (1.1)-(1.2), with $n \geq 2$ and the initial manifold $\mathscr{M}_{0}$ compact and of positive mean curvature. Then, for any $\eta>0$ there exists a constant $C_{\eta}>0$, depending only on $n, \eta$ and $\mathscr{M}_{0}$ such that

$$
R \geq-\eta H^{2}-C_{\eta}
$$

on $\mathscr{C l}_{t}$ for any $t \in[0, T[$.

Roughly speaking, this result shows that the negative part of the scalar curvature cannot grow as fast as $H^{2}$ when the latter tends to infinity. Following the ideas of Hamilton [7] and Huisken [10] one is led to look for an upper bound for the function $\left(|A|^{2}-H^{2}\right) / H^{2-\sigma}$ for some small positive $\sigma$. However, the method of [10] does not carry on to the present context, because it relies on some estimates which hold only for convex surfaces. Therefore we have to introduce an additional parameter $\eta$ and to study the function $g_{\sigma, \eta}$ defined in (2.2). Exploiting the presence of this parameter we obtain some inequalities (see Lemma 3.2) which allows us to bound $g_{\sigma, \eta}$ under the only hypothesis of positive mean curvature. This bound will yield Theorem 3.1.

The following estimate will be used in the proof of our theorem; we remark that it does not depend on our assumptions on $\mathscr{U}_{t}$ and is valid for any smooth hypersurface.

Lemma 3.2. Suppose $(1+\eta) H^{2} \leq|A|^{2} \leq c_{0} H^{2}$ for some $\eta, c_{0}>0$ at some point of $\mathscr{M}_{t}$. Then we also have 
(i) $-2 Z \geq \eta H^{2}|A|^{2}$;

(ii) $\left|H \nabla_{i} h_{k l}-\nabla_{i} H h_{k l}\right|^{2} \geq \frac{\eta^{2}}{4 n(n-1)^{2} c_{0}} H^{2}|\nabla H|^{2}$.

Proof. (i) Taking into account Lemma 2.2 and the assumption $|A| \geq \sqrt{1+\eta}|H|$ we obtain

$$
\begin{aligned}
-2 Z & =2\left(|A|^{4}-\operatorname{tr}\left(A^{3}\right) H\right) \geq 2|A|^{3}(|A|-|H|) \\
& \geq 2(1+\eta-\sqrt{1+\eta})|A|^{2} H^{2} \\
& \geq \eta|A|^{2} H^{2} .
\end{aligned}
$$

(ii) We have (see [10, Lemma 2.3(ii)])

$$
\begin{aligned}
\left|H \nabla_{i} h_{k l}-\nabla_{i} H h_{k l}\right|^{2} & \geq \frac{1}{4}\left|\nabla_{i} H h_{k l}-\nabla_{k} H h_{i l}\right|^{2} \\
& =\frac{1}{2}\left(|A|^{2}|\nabla H|^{2}-\left|\nabla^{i} H h_{i l}\right|^{2}\right) .
\end{aligned}
$$

Let us denote with $\lambda_{1}, \ldots, \lambda_{n}$ the eigenvalues of $A$ in such a way that $\lambda_{n}$ is an eigenvalue with the largest modulus. Then we have $\left|\nabla^{i} H h_{i l}\right|^{2} \leq \lambda_{n}^{2}|\nabla H|^{2}$ and

$$
\begin{aligned}
\left|H \nabla_{i} h_{k l}-\nabla_{i} H h_{k l}\right|^{2} & \geq \frac{1}{2} \sum_{i=1}^{n-1} \lambda_{i}^{2}|\nabla H|^{2}=\sum_{i=1}^{n-1} \lambda_{i}^{2} \lambda_{n}^{2} \frac{|\nabla H|^{2}}{2 \lambda_{n}^{2}} \\
& \geq \sum_{i=1}^{n-1} \sum_{j=i+1}^{n} \lambda_{i}^{2} \lambda_{j}^{2} \frac{|\nabla H|^{2}}{2(n-1)|A|^{2}} \\
& \geq\left(\sum_{\substack{i, j=1 \\
i<j}}^{n} \lambda_{i} \lambda_{j}\right)^{2} \frac{|\nabla H|^{2}}{n(n-1)^{2}|A|^{2}} \\
& =\frac{\left(|A|^{2}-H^{2}\right)^{2}}{4 n(n-1)^{2}|A|^{2}}|\nabla H|^{2} \geq \frac{\eta^{2} H^{2}}{4 n(n-1)^{2} c_{0}}|\nabla H|^{2} .
\end{aligned}
$$

Let us now consider the function $g_{\sigma, \eta}$ defined in (2.2). We assume that $\sigma, \eta \in$ ] $0,1\left[\right.$. In the following we denote by $c_{i}$ any positive constant depending only on $n, \eta$ and $\mathscr{C}_{0}$, and we assume that these constants are chosen larger than 1. We shall write for simplicity $g_{\sigma, \eta}=g$ as long as $\sigma, \eta$ are kept fixed. We observe that (2.5) implies

$$
g_{\sigma, \eta} \leq c_{0} H^{\sigma} .
$$

Remark 3.3. Let $g_{+}$denote the positive part of $g$, i.e. $g_{+}(x, t)=\max \{g(x, t), 0\}$. Then it is easily seen that $g_{+}^{p} \in C^{1}(\mathscr{L} \times[0, T[)$ for any $p>1$ and

$$
\frac{\partial g_{+}^{p}}{\partial t}=p g_{+}^{p-1} \frac{\partial g}{\partial t}, \quad \nabla\left(g_{+}^{p}\right)=p g_{+}^{p-1} \nabla g .
$$


Lemma 3.4. There exist constants $c_{2}, c_{3}$ such that

$$
\begin{aligned}
\frac{d}{d t} \int_{\mathscr{M}_{t}} g_{+}^{p} d \mu \leq & -\frac{p(p-1)}{2} \int_{\mathscr{M}_{t}} g_{+}^{p-2}|\nabla g|^{2} d \mu-\frac{p}{c_{3}} \int_{\mathscr{M}_{t}} \frac{g_{+}^{p-1}}{H^{2-\sigma}}|\nabla H|^{2} d \mu \\
& -p \int_{\mathscr{M}_{t}} \frac{g_{+}^{p-1}}{H^{4-\sigma}}\left|H \nabla_{i} h_{k l}-\nabla_{i} H h_{k l}\right|^{2} d \mu+p \sigma \int_{\mathscr{M}_{t}}|A|^{2} g_{+}^{p} d \mu
\end{aligned}
$$

for any $p \geq c_{2}$

Proof. By Remark 3.3, Proposition 2.1(iii) and equation (2.3) we have for $p \geq 2$

$$
\begin{aligned}
\frac{d}{d t} \int g_{+}^{p} d \mu= & \int\left(\frac{\partial g_{+}^{p}}{\partial t}-H^{2} g_{+}^{p}\right) d \mu \\
= & \int\left(p g_{+}^{p-1} \frac{\partial g}{\partial t}-H^{2} g_{+}^{p}\right) d \mu \\
\leq & -p(p-1) \int g_{+}^{p-2}|\nabla g|^{2} d \mu+2(1-\sigma) p \int \frac{g_{+}^{p-1}}{H}\langle\nabla H, \nabla g\rangle d \mu \\
& -2 p \int \frac{g_{+}^{p-1}}{H^{4-\sigma}}\left|H \nabla_{i} h_{k l}-\nabla_{i} H h_{k l}\right|^{2} d \mu+p \sigma \int|A|^{2} g_{+}^{p} d \mu .
\end{aligned}
$$

From Lemma 3.2(ii) and inequality (3.1) we deduce, if $c_{1} \geq 4 n(n-1)^{2} c_{0} \eta^{-2}$,

$$
\begin{aligned}
\frac{g_{+}^{p-1}}{H^{4-\sigma}}\left|H \nabla_{i} h_{k l}-\nabla_{i} H h_{k l}\right|^{2} & \geq \frac{g_{+}^{p-1}}{c_{1} H^{2-\sigma}}|\nabla H|^{2} \\
& \geq \frac{g_{+}^{p-1}}{2 c_{1} H^{2-\sigma}}|\nabla H|^{2}+\frac{1}{2 c_{0} c_{1}} \frac{g_{+}^{p}}{H^{2}}|\nabla H|^{2}
\end{aligned}
$$

Therefore, if $p \geq \max \left\{2,1+4 c_{0} c_{1}\right\}$ we obtain

$$
\begin{aligned}
& 2(1-\sigma) p \frac{g_{+}^{p-1}}{H}\langle\nabla H, \nabla g\rangle \\
\leq & 2 p \frac{g_{+}^{p-1}}{H}|\nabla H||\nabla g| \\
\leq & \frac{p}{2 c_{0} c_{1}} \frac{g_{+}^{p}}{H^{2}}|\nabla H|^{2}+2 c_{0} c_{1} p g_{+}^{p-2}|\nabla g|^{2} \\
\leq & p \frac{g_{+}^{p-1}}{H^{4-\sigma}}\left|H \nabla_{i} h_{k l}-\nabla_{i} H h_{k l}\right|^{2}+\frac{p(p-1)}{2} g_{+}^{p-2}|\nabla g|^{2} \\
& -p \frac{g_{+}^{p-1}}{2 c_{1} H^{2-\sigma}}|\nabla H|^{2} .
\end{aligned}
$$

Substituting in inequality (3.2) we obtain the conclusion.

Lemma 3.5. There exists a constant $c_{4}$ such that 


$$
\begin{aligned}
& \frac{1}{c_{4}} \int_{\mathscr{U}_{t}}|A|^{2} g_{+}^{p} d \mu \\
\leq & \left(p+\frac{p}{\beta}\right) \int_{\mathscr{M}_{t}} g_{+}^{p-2}|\nabla g|^{2} d \mu+(1+\beta p) \int_{\mathscr{M}_{t}} \frac{g_{+}^{p-1}}{H^{2-\sigma}}|\nabla H|^{2} d \mu \\
& +\int_{\mathscr{M}_{t}} \frac{g_{+}^{p-1}}{H^{4-\sigma}}\left|H \nabla_{i} h_{k l}-\nabla_{i} H h_{k l}\right|^{2} d \mu
\end{aligned}
$$

for any $\beta>0, p>2$.

Proof. Let us set $\alpha=2-\sigma$. We compute

$$
\begin{aligned}
\Delta g= & \Delta\left(\frac{|A|^{2}}{H^{\alpha}}\right)-(1+\eta) \Delta H^{2-\alpha} \\
= & \frac{\Delta|A|^{2}}{H^{\alpha}}-\alpha \frac{|A|^{2}}{H^{\alpha+1}} \Delta H-\alpha(\alpha-1) \frac{|A|^{2}}{H^{\alpha+2}}|\nabla H|^{2} \\
& -\frac{2 \alpha}{H}\left\langle\nabla H, \nabla \frac{|A|^{2}}{H^{\alpha}}\right\rangle+(1+\eta)(2-\alpha)\left(\frac{\alpha-1}{H^{\alpha}}|\nabla H|^{2}-\frac{1}{H^{\alpha-1}} \Delta H\right) .
\end{aligned}
$$

Using formula (2.6) can rewrite the above equality as

$$
\begin{aligned}
\Delta g= & \frac{2}{H^{\alpha}}\left\langle h_{i j}, \nabla_{i} \nabla_{j} H\right\rangle+\frac{2}{H^{\alpha}} Z+\frac{2}{H^{\alpha+2}}\left|H \nabla_{i} h_{k l}-\nabla_{i} H h_{k l}\right|^{2} \\
& -\left(\frac{\alpha g}{H}+2(1+\eta) H^{1-\alpha}\right) \Delta H+\frac{(2-\alpha)(\alpha-1)}{H^{2}} g|\nabla H|^{2} \\
& -\frac{2(\alpha-1)}{H}\langle\nabla H, \nabla g\rangle .
\end{aligned}
$$

We multiply this equality by $g_{+}^{p} H^{\alpha-2}$ and integrate on $\mathscr{M}_{t}$. Taking into account Remark 3.3 and the Codazzi equation we can integrate by parts and obtain

$$
\begin{aligned}
& \int-\frac{2 Z}{H^{2}} g_{+}^{p} d \mu \\
= & p \int \frac{g_{+}^{p-1}}{H^{2-\alpha}}|\nabla g|^{2} d \mu-2 p \int \frac{g_{+}^{p-1}}{H^{2}}\left\langle h_{i j}, \nabla_{i} g \nabla_{j} H\right\rangle d \mu \\
& +4 \int \frac{g_{+}^{p}}{H^{3}}\left\langle h_{i j}, \nabla_{i} H \nabla_{j} H\right\rangle d \mu+2 \int \frac{g_{+}^{p}}{H^{4}}\left|H \nabla_{i} h_{k l}-\nabla_{i} H h_{k l}\right|^{2} d \mu \\
& +\int\left(\alpha p \frac{g_{+}^{p}}{H^{3-\alpha}}+2 p(1+\eta) \frac{g_{+}^{p-1}}{H}\right)\langle\nabla g, \nabla H\rangle d \mu \\
& -2 \int\left(\frac{g_{+}^{p+1}}{H^{4-\alpha}}+(2+\eta) \frac{g_{+}^{p}}{H^{2}}\right)|\nabla H|^{2} d \mu .
\end{aligned}
$$

Using repeatedly inequality (3.1) and the assumptions $\alpha \in] 1,2[, \eta \in] 0,1[$ we obtain 


$$
\begin{aligned}
\int-\frac{2 Z}{H^{2}} g_{+}^{p} d \mu \leq & c_{0} p \int g_{+}^{p-2}|\nabla g|^{2} d \mu \\
& +4 p\left(c_{0}+1\right) \int \frac{g_{+}^{p-1}}{H}|\nabla H||\nabla g| d \mu \\
& +4 c_{0}^{2} \int \frac{g_{+}^{p-1}}{H^{\alpha}}|\nabla H|^{2} d \mu+2 c_{0} \int \frac{g_{+}^{p-1}}{H^{2+\alpha}}\left|H \nabla_{i} h_{k l}-\nabla_{i} H h_{k l}\right|^{2} d \mu .
\end{aligned}
$$

In addition we have, for any $\beta>0$

$$
\begin{aligned}
2 \frac{g_{+}^{p-1}}{H}|\nabla H||\nabla g| & \leq \frac{g_{+}^{p-2}}{\beta}|\nabla g|^{2}+\beta \frac{g_{+}^{p}}{H^{2}}|\nabla H|^{2} \\
& \leq \frac{g_{+}^{p-2}}{\beta}|\nabla g|^{2}+c_{0} \beta \frac{g_{+}^{p-1}}{H^{\alpha}}|\nabla H|^{2} .
\end{aligned}
$$

The assertion follows from the above inequalities and from Lemma 3.2(i).

Proposition 3.6. Given $\eta \in] 0,1\left[\right.$, there exist constants $c_{5}$, $c_{6}$ such that the $L^{p}(\mathscr{C})$ norm of $\left(g_{\sigma, \eta}\right)_{+}$is a nonincreasing function of $t$ for any $p, \sigma$ such that

$$
p \geq c_{5}, \quad \sigma \leq\left(c_{6} p\right)^{-1 / 2} .
$$

Proof. Let $c_{2}, c_{3}, c_{4}$ be as in Lemmas 3.4, 3.5. We recall that we assumed to choose all these constants greater than 1 . Suppose that $p, \sigma$ satisfy

$$
p \geq \max \left\{4, c_{2}, \frac{c_{3}}{2}\right\}, \quad \sigma \leq \frac{1}{c_{4}} \sqrt{\frac{1}{8 c_{3} p}} .
$$

If we set $\beta=4 c_{4} \sigma$, then

$$
\left\{\begin{array}{l}
p^{2} \sigma c_{4}\left(\beta+\frac{1}{\beta}\right)=\frac{p^{2}}{4}\left(\beta^{2}+1\right) \leq \frac{p^{2}}{4}+\frac{p}{2} \leq \frac{p(p-1)}{2} \\
p \sigma c_{4}(1+\beta p) \leq \sqrt{\frac{p}{8 c_{3}}}\left(1+\sqrt{\frac{2 p}{c_{3}}}\right) \leq 2 \sqrt{\frac{p}{8 c_{3}}} \sqrt{\frac{2 p}{c_{3}}}=\frac{p}{c_{3}} \\
p \sigma c_{4} \leq \sqrt{\frac{p}{8 c_{3}}} \leq p
\end{array}\right.
$$

Thus, by Lemma 3.5,

$$
\begin{aligned}
p \sigma \int|A|^{2} g_{+}^{p} d \mu \leq & \frac{p(p-1)}{2} \int g_{+}^{p-2}|\nabla g|^{2} d \mu+\frac{p}{c_{3}} \int \frac{g_{+}^{p-1}}{H^{2-\sigma}}|\nabla H|^{2} d \mu \\
& +p \int \frac{g_{+}^{p-1}}{H^{4-\sigma}}\left|H \nabla_{i} h_{k l}-\nabla_{i} H h_{k l}\right|^{2} d \mu .
\end{aligned}
$$

By Lemma 3.4 we conclude

$$
\frac{d}{d t} \int g_{+}^{p} d \mu \leq 0
$$


Proof of Theorem 3.1. From the $L^{p}$-estimate of the previous Proposition one can derive a uniform bound on the supremum of $g_{\sigma, \eta}$ with the procedure of [10, Theorem 5.1]. We give a sketch of this method for the reader's convenience. Given any $k \geq k_{0}$, where

$$
k_{0}:=\sup _{\sigma \in[0,1] \mathscr{M}_{0}} \sup _{0} g
$$

we set

$$
v=\left(g_{\sigma, \eta}-k\right)_{+}^{p / 2}, \quad A(k, t)=\left\{x \in \mathscr{M}_{t}: v(x, t)>0\right\} .
$$

Arguing as in the proof of Lemma 3.4 we obtain, for $p$ large enough,

$$
\frac{d}{d t} \int v^{2} d \mu+\int|\nabla v|^{2} d \mu \leq c_{0} \sigma p \int_{A(k, t)} H^{2} g_{\sigma, \eta}^{p} d \mu .
$$

On the other hand we have the following Sobolev-type inequality (see [15]) valid for any Lipschitz function on $\mathscr{M}_{t}$ :

$$
\left(\int v^{2 q} d \mu\right)^{1 / q} \leq c_{7} \int|\nabla v|^{2} d \mu+c_{7}\left(\int_{A(k, t)} H^{n} d \mu\right)^{2 / n}\left(\int v^{2 q} d \mu\right)^{1 / q} .
$$

Here $q=n /(n-2)$ if $n>2$ and an arbitrary number greater than 1 if $n=2$. Now we observe that, if

$$
p \geq \max \left\{c_{5}, 4 n^{2} c_{6}\right\}, \quad \sigma \leq\left(4 c_{6} p\right)^{-1 / 2}
$$

then we have

$$
\int_{\mathscr{M}_{t}} H^{n} g_{\sigma, \eta}^{p} d \mu=\int_{\mathscr{M}_{t}} g_{\sigma^{\prime}, \eta}^{p} d \mu
$$

with

$$
\sigma^{\prime}=\sigma+\frac{n}{p} \leq \frac{1}{2 \sqrt{c_{6} p}}+\frac{1}{\sqrt{p}} \frac{n}{\sqrt{p}} \leq \frac{1}{\sqrt{c_{6} p}} .
$$

Thus, by Proposition 3.6,

$$
\begin{aligned}
\left(\int_{A(k, t)} H^{n} d \mu\right)^{2 / n} & \leq k^{-2 p / n}\left(\int_{A(k, t)} H^{n} g_{\sigma, \eta}^{p} d \mu\right)^{2 / n} \\
& \leq k^{-2 p / n}\left(\int_{\mathscr{M}_{0}} g_{\sigma^{\prime}, \eta}^{p} d \mu\right)^{2 / n} \\
& \leq\left(\frac{\left(1+\left|\mathscr{M}_{0}\right|\right) k_{0}}{k}\right)^{2 p / n}
\end{aligned}
$$

Thus, we can fix $k_{1}>k_{0}$ large enough such that, for any $k \geq k_{1}$ we may absorb the last term in (3.4) and then exploit the $|\nabla v|$ term in (3.3) to obtain

$$
\frac{d}{d t} \int v^{2} d \mu+\frac{1}{c_{8}}\left(\int v^{2 q} d \mu\right)^{1 / q} \leq c_{0} \sigma p \int_{A(k, t)} H^{2} g_{\sigma, \eta}^{p} d \mu .
$$


After a time integration and a computation involving the Hölder inequality and the interpolation inequality for $L^{p}$ spaces (see [10] for details) we obtain, for any $r>1$,

$$
\begin{aligned}
& \int_{0}^{T} \int_{A(k, t)} v^{p} d \mu d t \\
& \leq c_{9} \sigma p\left(\int_{0}^{T} \int_{A(k, t)} d \mu d t\right)^{1+b-1 / r}\left(\int_{0}^{T} \int_{A(k, t)} H^{2 r} g_{\sigma, \eta}^{p r} d \mu d t\right)^{1 / r}
\end{aligned}
$$

where $b=(q-1) /(2 q-1)$. Let us now choose $r$ large enough such that $\gamma:=1+b-1 / r>1$. With an argument as in (3.5) we can estimate the second factor on the right hand side provided $p, \sigma^{-1}$ are larger than suitable constants depending only on $n, \eta, \mathscr{M}_{0}$. Thus we find a constant $c_{10}$ such that, for all $h>k \geq k_{1}$,

$$
\begin{aligned}
|h-k|^{p} \int_{0}^{T} \int_{A(h, t)} d \mu d t & \leq \int_{0}^{T} \int_{A(k, t)} v^{p} d \mu d t \\
& \leq c_{10}^{p} \sigma p\left(\int_{0}^{T} \int_{A(k, t)} d \mu d t\right)^{\gamma}
\end{aligned}
$$

for all $h>k \geq k_{1}$. By a well-known result (see e.g. [16, Lemma 4.1]) we conclude

$$
\int_{0}^{T} \int_{A(k, t)} d \mu d t=0, \quad \forall k>k_{1}+d^{1 / p},
$$

where

$$
d=c_{10}^{p} \sigma p 2^{p \gamma /(\gamma-1)}\left(\int_{0}^{T} \int_{A\left(k_{1}, t\right)} d \mu d t\right)^{\gamma-1}
$$

Here we use the properties that the critical time $T$ is finite and that the area of $\mathscr{M}_{t}$ decreases with $t$, which follow from (2.1) and Proposition 2.1(iii) respectively. We obtain, by the definition of $A(k, t)$,

$$
|A|^{2} \leq(1+\eta) H^{2}+\left(k_{1}+d^{1 / p}\right) H^{2-\sigma} .
$$

This implies that

$$
|A|^{2} \leq(1+2 \eta) H^{2}+K_{\eta}
$$

for some $K_{\eta}$ depending only on $\eta, n, \mathscr{C}_{0}$. Setting $C_{\eta}=K_{\eta / 2}$ we conclude that $|A|^{2}-H^{2} \leq \eta H^{2}+C_{\eta}$, which proves the theorem.

\section{Asymptotic behaviour}

We now apply the estimate of the previous section to study the asymptotic behaviour of our family of hypersurfaces near a singularity. It is customary to divide the singularities in two classes, depending on the rate at which the curvature becomes unbounded. 
Definition 4.1. We say that our surface develops a singularity of type I (or a fast singularity) as $t \rightarrow T$ if there exists a constant $C>0$ such that

$$
\max _{\mathscr{C O}_{t}}|A|^{2} \leq \frac{C}{T-t}
$$

Otherwise we say that the singularity is of type II (or slow).

Remark 4.2. (i) By a comparison argument using the evolution equation for $|A|^{2}$ (see Proposition 2.1(ii)) it is easily seen that $\max _{\mathscr{A G}_{t}}|A|^{2} \geq[2(T-t)]^{-1}$.

(ii) From inequality (2.5) and from the general property $H^{2} \leq n|A|^{2}$ we deduce that under our hypotheses $H^{2}$ and $|A|^{2}$ have the same blow-up rate.

To study the shape of the surface near a singularity we define now a family of rescaled flows. Since singularities of type I have already been classified in [11], [12], we consider the case of type II singularities. Following an idea of Hamilton [8], we choose a sequence $\left\{\left(x_{k}, t_{k}\right)\right\}$ as follows. For any integer $k \geq 1$ let $t_{k} \in[0, T-1 / k], x_{k} \in \mathscr{M} 6$ be such that

$$
H^{2}\left(x_{k}, t_{k}\right)\left(T-\frac{1}{k}-t_{k}\right)=\max _{\substack{t \leq T-1 / k \\ x \in \mathscr{M}}} H^{2}(x, t)\left(T-\frac{1}{k}-t\right) .
$$

Furthermore we set

$$
L_{k}=H\left(x_{k}, t_{k}\right), \quad \alpha_{k}=-L_{k}^{2} t_{k}, \quad \omega_{k}=L_{k}^{2}\left(T-t_{k}-1 / k\right) .
$$

Lemma 4.3. If the singularity is of type II then we have, as $k \rightarrow \infty$,

$$
t_{k} \rightarrow T, \quad L_{k} \rightarrow \infty, \quad \alpha_{k} \rightarrow-\infty, \quad \omega_{k} \rightarrow \infty .
$$

Proof. Let $M>0$ be given. Since we are assuming that the singularity is of type II (see also Remark 4.2(ii)) there exist $\bar{t}<T$ and $\bar{x} \in \mathscr{M}$ such that $H^{2}(\bar{x}, \bar{t})(T-\bar{t})>2 M$. For $k$ large enough we have

$$
\bar{t}<T-1 / k, \quad H^{2}(\bar{x}, \bar{t})(T-\bar{t}-1 / k)>M .
$$

It follows

$$
\omega_{k}=H^{2}\left(x_{k}, t_{k}\right)\left(T-t_{k}-1 / k\right) \geq H^{2}(\bar{x}, \bar{t})(T-\bar{t}-1 / k)>M .
$$

Since $\omega_{k}$ is increasing and $M>0$ is arbitrary, this proves that $\omega_{k} \rightarrow \infty$. Furthermore, it follows from the definitions that

$$
\omega_{k} \rightarrow \infty \quad \Rightarrow \quad L_{k} \rightarrow \infty \quad \Rightarrow \quad t_{k} \rightarrow T, \alpha_{k} \rightarrow-\infty
$$

Now consider, for any $k \geq 1$, the family of surfaces $\mathscr{M}_{k, \tau}$ defined by the immersions

$$
F_{k}(\cdot, \tau)=L_{k}\left(F\left(\cdot, L_{k}^{-2} \tau+t_{k}\right)-F\left(x_{k}, t_{k}\right)\right), \quad \tau \in\left[\alpha_{k}, \omega_{k}\right] .
$$

We denote by $A_{k}$ and $H_{k}$ the second fundamental form and the mean curvature associated with the immersion $F_{k}$. 
Lemma 4.4. If the singularity is of type II then the rescaled immersions satisfy the following properties:

(i) $F_{k}\left(x_{k}, 0\right)=0, H_{k}\left(x_{k}, 0\right)=1$

(ii) for any $\eta>0$ there exists $C_{\eta}$ such that $\left|A_{k}\right|^{2} \leq(1+\eta) H_{k}^{2}+C_{\eta} L_{k}^{-2}$;

(iii) for any $\varepsilon>0$ and $\bar{\omega}>0$ there exists $k_{0}$ such that $\max H_{k}(\cdot, \tau) \leq 1+\varepsilon$ for any $k \geq k_{0}, \tau \in\left[\alpha_{k_{0}}, \bar{\omega}\right]$.

Proof. We have by the definition of $F_{k}$

$$
A_{k}(x, \tau)=L_{k}^{-1} A\left(x, L_{k}^{-2} \tau+t_{k}\right) .
$$

From this we deduce (i) and (ii), by virtue of Theorem 3.1. In addition, our choice of $\left(x_{k}, t_{k}\right)$ implies

$$
H_{k}^{2}(x, \tau) \leq \frac{T-\frac{1}{k}-t_{k}}{T-\frac{1}{k}-t_{k}-L_{k}^{2} \tau}=\frac{\omega_{k}}{\omega_{k}-\tau}
$$

for any $k$ and $\tau \in\left[\alpha_{k}, \omega_{k}\left[\right.\right.$. Since $\omega_{k} \rightarrow \infty$ we obtain (iii).

Theorem 4.5. Let $\mathscr{M}_{t}, t \in[0, T$ [ be a smooth solution of the mean curvature flow (1.1)-(1.2), with $n \geq 2$. Assume that the initial manifold $\mathscr{M}_{0}$ is compact and of positive mean curvature, and that the flow develops a singularity of type II as $t \rightarrow T$. Then a subsequence of the flows $\mathscr{C l}_{k, \tau}$ converges smoothly to a mean curvature flow $\widetilde{\mathbb{M}_{\tau}}$, defined for $\tau \in \mathbb{R}$. The mean curvature $\widetilde{H}$ of the limit flow satisfies $0<\widetilde{H} \leq 1$ and is equal to 1 at least at one point. Furthermore, either $\mathscr{\mathscr { O }}_{\tau}$ has positive scalar curvature everywhere or (up to a rigid motion) $\mathscr{\mathscr { O }}_{\tau}=$ $\mathbb{R}^{n-1} \times \bar{\Gamma}_{\tau}$, where $\bar{\Gamma}_{\tau}$ is the "grim reaper" curve given by $x=-\ln \cos y+\tau$.

Proof. The curvature bound in (iii) of the previous lemma implies analogous bounds on the second fundamental form and all its covariant derivatives. Then by standard methods, based on the Arzelà-Ascoli theorem, we can extract a subsequence of the $\mathscr{\mathscr { C }}_{k, \tau}$ converging uniformly on compact subsets of $\mathbb{R}^{n+1} \times \mathbb{R}$ to a limiting solution $\widetilde{\mathscr{C}}_{\tau}$ of the mean curvature flow, whose mean curvature satisfies $\widetilde{H} \leq 1$. Since $0 \in \mathscr{N}_{k, 0}$ for any $k$ and $H_{k}=1$ at that point, the same holds for the limit flow. Being the limit of surfaces with positive mean curvature, the limit flow has nonnegative mean curvature. But the maximum principle implies that $\widetilde{H}$ is actually everywhere positive, since it satisfies the equation in Proposition 2.1(i) and is not identically zero.

Furthermore, the estimate in Lemma 4.4(ii) implies that $|\widetilde{A}|^{2} \leq \widetilde{H}^{2}$. Again the maximum principle, together with equation (2.4), implies that either $|\widetilde{A}|^{2}<\widetilde{H}^{2}$ everywhere (i.e. the limiting surface has positive scalar curvature), or

$$
|\widetilde{A}|^{2} \equiv \widetilde{H}^{2}, \quad\left|\widetilde{H} \nabla_{i} \widetilde{h}_{k l}-\nabla_{i} \widetilde{H} \widetilde{h}_{k l}\right|^{2} \equiv 0 .
$$

In the latter case, arguing as in [12, Theorem 5.1], we obtain that (up to rigid motions) $\widetilde{\mathscr{C l}_{\tau}}=\mathbb{R}^{n-1} \times \Gamma_{\tau}$, where $\Gamma_{\tau}$ is a convex eternal solution to the mean curvature flow in the plane, whose mean curvature assumes its maximum at at 
least one point. By Hamilton's characterization of convex eternal solutions [9, Theorem 1.3] we deduce that $\Gamma_{\tau}$ is a translating soliton, and it is well known that the only translating soliton in the plane is the grim reaper.

Remark 4.6. A similar procedure can be followed in the case of a surface developing a singularity of type I. In this case we choose a sequence $\left\{\left(x_{k}, t_{k}\right)\right\}$ such that $t_{k} \rightarrow T$ and $H\left(x_{k}, t_{k}\right)=\max _{16} H\left(\cdot, t_{k}\right)$. Then we define the rescaled flows as in (4.3)-(4.4). In this case we obtain convergence to a limit flow $\widetilde{\mathscr{C O}_{\tau}}$ defined in a maximal interval of the form ] $-\infty, \Omega$ [ for some $\Omega<+\infty$. The limit flow has again nonnegative scalar curvature; however for singularities of type I this property follows already from the selfsimilarity result in [11, Theorem 3.5] and the classification in [12, Theorem 5.1].

For the two-dimensional case we now obtain a detailed description of possible singular behaviour.

Corollary 4.7. Suppose $F_{t}: \mathscr{l}^{2} \rightarrow \mathbb{R}^{3}$ is a smooth solution of (1.1)-(1.2) compact and with positive mean curvature on the maximal time interval [0,T [.

(i) If the singularity for $t \rightarrow T$ is of type $I$, the only possible limiting flows under the rescaling procedure (4.4) are the homothetically shrinking solutions associated with $S^{2}, \mathbb{R} \times S^{1}$ and $\mathbb{R} \times \Gamma$, where $\Gamma$ is one of the selfsimilar immersed curves introduced by Mullins [13] (see also Abresch-Langer [1]).

(ii) If the singularity is of type II, then the limiting flow is either a strictly convex translating soliton or the translating solution given by $\mathbb{R} \times \bar{\Gamma}_{\tau}$, where $\bar{\Gamma}_{\tau}$ is the "grim reaper" curve given by $x=-\ln \cos y+\tau$.

Proof. The first part is a consequence of the results quoted in Remark 4.6. The second part follows from Theorem 4.5 and Hamilton's result [9] characterizing convex eternal solutions, since for $n=2$ positive scalar curvature is equivalent to convexity.

Acknowledgements. This research was done while the second author was visiting the University of Tübingen, whose hospitality he gratefully acknowledges.

\section{References}

1. U. Abresch, J. Langer, The normalized curve shortening flow and homothetic solutions, J. Differential Geom. 23 (1986), 175-196.

2. S. Altschuler, S.B. Angenent, Y. Giga, Mean curvature flow through singularities for surfaces of rotation, J. Geom. Analysis 5 (1995), 293-358.

3. S.B. Angenent, J.J.L. Velazquez, Degenerate neckpinches in mean curvature flow, J. Reine Angew. Math. 482 (1997), 15-66

4. K.A. Brakke, The motion of a surface by its mean curvature, Math. Notes, Princeton University Press, Princeton, NJ, 1978.

5. M. Gage, R.S. Hamilton, The shrinking of convex plane curves by the heat equation, J. Differential Geom. 23 (1986), 69-96.

6. M.A. Grayson, The heat equation shrinks embedded plane curves into points, J. Differential Geom. 26 (1987), 285-314. 
7. R.S. Hamilton, Three-manifolds with positive Ricci curvature, J. Differential Geom. 17 (1982), 255-306.

8. R.S. Hamilton, The formation of singularities in the Ricci Flow, Surveys in Differential Geometry Vol. II, International Press, Cambridge MA (1993), 7-136.

9. R.S. Hamilton, Harnack estimate for the mean curvature flow, J. Differential Geom. 41 (1995), 215-226.

10. G. Huisken, Flow by mean curvature of convex surfaces into spheres, J. Diff. Geometry 20 (1984), 237-266.

11. G. Huisken, Asymptotic behaviour for singularities of the mean curvature flow, J. Diff. Geometry 31 (1990), 285-299.

12. G. Huisken, Local and global behaviour of hypersurfaces moving by mean curvature, Proceedings of Symposia in Pure Mathematics 54 (1993), 175-191.

13. W. Mullins, Two dimensional motion of idealised grain boundaries, J. Appl. Physics 27 (1956), 900-904.

14. T. Ilmanen, Singularities of mean curvature flow of surfaces, preprint, Northwestern University .

15. J. H. Michael, L. M. Simon, Sobolev and mean value inequalities on generalized submanifolds of $\mathbb{R}^{n}$, Comm. Pure Appl. Math. 26 (1973), 361-379.

16. G. Stampacchia, Equations elliptiques au second ordre à coéfficients discontinues, Sém. Math. Sup. 16, Les Presses de l'Université de Montreal, Montreal, 1966.

17. B. White, Stratification of minimal surfaces, mean curvature flows, and harmonic maps, J. Reine Angew. Math. 488 (1997), 1-35. 